\title{
A Ge/SiNx Standard for Evaluating the Performance of X-ray Detectors in the SEM, S/TEM and AEM
}

\author{
Nestor J. Zaluzec ${ }^{1}$, Jon-Paul DesOrmeaux ${ }^{2}$, James Roussie ${ }^{2}$, \\ ${ }^{1 .}$ Electron Microscopy Center, Center for Nanoscale Materials, Argonne National Laboratory, Argonne, \\ IL, USA \\ 2. TEMwindows /SiMPore Inc, West Henrietta NY USA
}

With the rapidly evolving geometries of the current generation of X-ray detectors interfaced to all forms of electron optical columns, there arises an important need to characterize and compare these detector systems quantitatively. A comprehensive protocol requires not only a well defined methodology to insure that comparisons are made on equal footing between systems [1-3] but also a well characterized "test specimen". This is particularly important when addressing issues such as absolute quantification and/or absorption corrections for compositional measurements as well as the emerging applications, such as 3D elemental tomography [4]. An ideal test specimen in addition to being uniform and robust, would also facilitate characterization of the XEDS system in the following areas:

\section{Detector Energy Axis and Energy Resolution Calibration \\ Detector Window Transmission Evaluation \\ Detector Solid Angle Measurements \\ Electron Optical Instrument System Peak Measurements \\ Specimen Holder Penumbra Measurements}

Two recent studies have proposed either thin film amorphous $\mathrm{Ge}$ [2] or Bulk $\mathrm{Cu}$ [4] as a candidate test specimen, both of which have merits as well as limitations. Self supporting ultra thin Ge films are versatile and can address the an extensive array of measurements, however, handling and fabrication of large quantities for distribution is difficult. Bulk $\mathrm{Cu}$ is a routinely available material but is inappropriate for the TEM. In this work we have evaluated a hybrid Ge/SiNx specimen that alleviates many of the issues which have plagued alternate proposals.

The candidate specimen has been fabricated by electron beam evaporation of $\sim 30 \mathrm{~nm}$ of amorphous Ge on to a $20 \mathrm{~nm}$ thick SiNx self supporting window supported upon a $100 \mu \mathrm{m}$ thick Si frame. Figure 1, shows a 100x100 $\mu \mathrm{m} \mathrm{Ge} / \mathrm{SiNx}$ window area with corresponding electron diffraction pattern. At spatial dimensions greater than the $100 \mathrm{~nm}$ level the film is essentially uniform, however, high resolution TEM imaging reveals that the Germanium layer consist of small interconnected islands each about 5-10 $\mathrm{nm}$ in size, separated by a small inter-island gap $\leq 0.5 \mathrm{~nm}$, occasional Ge debris is found on the film but does not affect the performance as it can be judiciously avoided. Figure 2 plots a compendium of 18

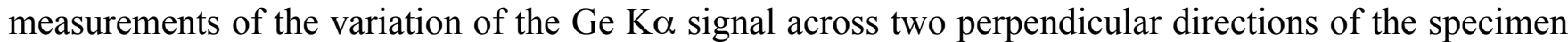
window, illustrating the uniformity of the signal. The measured integrated intensity variation of the $\mathrm{Ge}$ $\mathrm{K} \alpha$ Integral was $0.5 \%$, which is currently better than the accuracy to which the electron beam screen current could be monitored. Using the combination of the NK, Ge L, SiK and GeK peaks one can readily calibrate the detector energy axis and measure its energy resolution over a large energy range. Similarly, ratioing the $\mathrm{NK}, \mathrm{SiK}$, and GeL in comparison to the GeK gives an excellent measure to any window effects or deterioration due to detector contamination. Since Ge is not an element within the normal instrument fabrication it cannot be mistaken or confused with system peaks, and the regime in which system peaks normally occur [2-9 keV and 11-20 keV] is devoid of peaks from the Ge (figure 3 ). 
One of the most critical measurements in the TEM/AEM environment is identification of the penumbra of any specimen holder and this is readily accomplished [6]. Finally, using the procedures defined previously [1] together with measured values of the incident probe current and Ge layer thicknesses the solid angle of the detector can be measured.[2] Additional work is in progress to increase the window size to $\sim 500 \mu \mathrm{m}$ of the $\mathrm{SiNx}$ as well as to reduce the size of the Ge nano-islands and eliminate any residual gaps. [7]

\section{References:}

[1] N.J. Zaluzec Microsc. Microanal (2009) 15 (S2) 520.

[2] F. Schamberg Microsc. Microanal (2015), 21 (S3) 1479.

[3] N.J. Zaluzec, Microsc. Microanal (2013) 19 (S2) 1262.

[4] T. Slater et al , Ultramicroscopy (2016), 16261.

[5] N.J. Zaluzec Microsc. Microanaly. (2014), 20[4] 1318.

[6] N.J. Zaluzec Microsc. Microanaly. (2016) 22, these proceedings

[7] Research supported in part by U.S. DoE, Office of Science, Contract No. DE-AC02-06CH11357 in the Electron Microscopy Center of the Center for Nanoscale Materials at Argonne National Laboratory
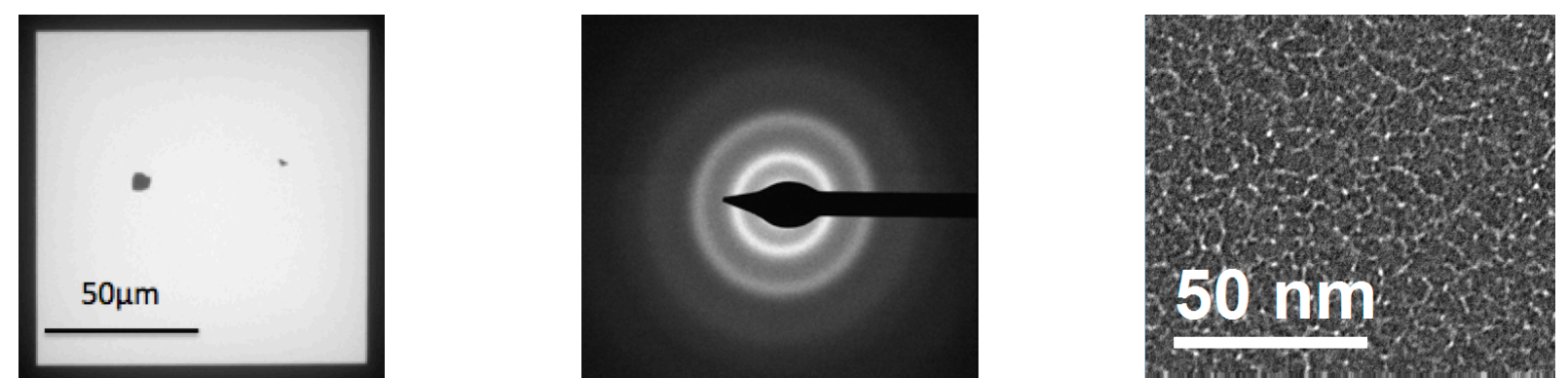

Figure 1. a) $100 \times 100 \mu \mathrm{m} \mathrm{Ge} / \mathrm{SiNx}$ window, notice random particle debris which does not affect the viability of the window or measurements thereupon, b) select area diffraction pattern confirming amorphous structure of the Ge/SiNx film c) Ge Islands visible at higher magnification with the interisland gaps $<1 \mathrm{~nm}$.

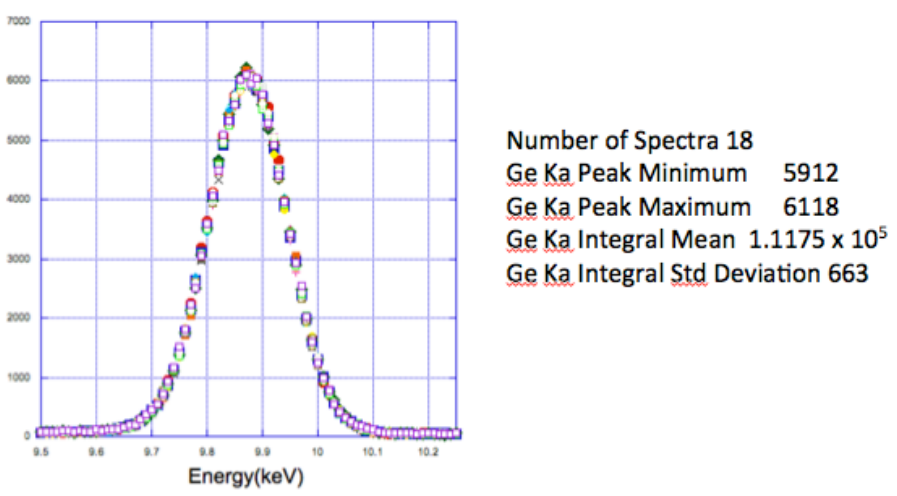

Figure 2. Reproducibility of Spectral Profiles across two perpendicular directions of the Ge/SiNx window

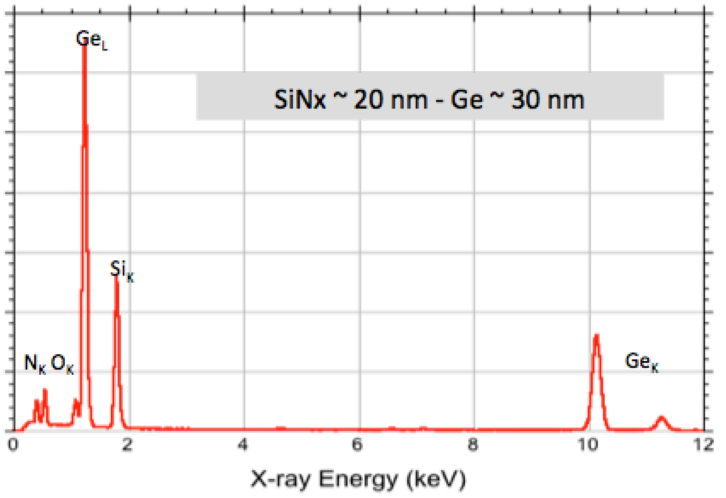

Figure 3. Typical Ge/SiNx spectral profile at $\sim 1 \mathrm{nA}$ and $200 \mathrm{kV}$. 